\title{
Evidence for Dominant Phonon-Electron Scattering in Weyl Semimetal WP
}

\author{
Gavin B. Osterhoudt $\odot,{ }^{1}$ Yaxian Wang $\odot,{ }^{2}$ Christina A. C. Garcia $\odot,{ }^{2}$ Vincent M. Plisson $\odot,{ }^{1}$ Johannes Gooth, ${ }^{3}$ \\ Claudia Felser $\odot,{ }^{3}$ Prineha Narang $\odot,^{2}$ and Kenneth S. Burch ${ }^{1, *}$ \\ ${ }^{1}$ Department of Physics, Boston College, Chestnut Hill, Massachusetts 02467, USA \\ ${ }^{2}$ John A. Paulson School of Engineering and Applied Sciences, Harvard University, \\ Cambridge, Massachusetts 02138, USA \\ ${ }^{3}$ Max Planck Institute for Chemical Physics of Solids, Dresden 01187, Germany
}

(Received 16 June 2020; revised 19 November 2020; accepted 2 December 2020; published 27 January 2021)

\begin{abstract}
Topological semimetals have revealed a wide array of novel transport phenomena, including electron hydrodynamics, quantum field theoretic anomalies, and extreme magnetoresistances and mobilities. However, the scattering mechanisms central to the fundamental transport properties remain largely unexplored. Here, we reveal signatures of significant phonon-electron scattering in the type-II Weyl semimetal $\mathrm{WP}_{2}$ via temperature-dependent Raman spectroscopy. Over a large temperature range, we find that the decay rates of the lowest energy $A_{1}$ modes are dominated by phonon-electron rather than phononphonon scattering. In conjunction with first-principles calculations, a combined analysis of the momentum, energy, and symmetry-allowed decay paths indicates this results from finite momentum interband and intraband scattering of the electrons. The excellent agreement with theory further suggests that such results could be true for the acoustic modes. We thus provide evidence for the importance of phonons in the transport properties of topological semimetals and identify specific properties that may contribute to such behavior in other materials.
\end{abstract}

DOI: 10.1103/PhysRevX.11.011017

Subject Areas: Condensed Matter Physics, Optics

\section{INTRODUCTION}

Topological semimetals display a range of novel transport phenomena, including enormous magnetoresistance and mobilities [1-5]. Observations of such behavior in topological systems suggest that the quantum geometry of the electronic bands is a crucial ingredient. However, nontopological semimetals such as LaAs, $\mathrm{PtSn}_{4}$, and $\alpha$ - $\mathrm{WP}_{2}$ have revealed similarly remarkable magnetoresistances and mobilities [6-8], suggesting that these properties are more endemic to semimetals, in general, with some works proposing that the primary factor is the near-perfect electron-hole compensation [6]. In addition, there is often enormous variation in the temperature dependence of these properties, indicating that temperature-dependent scattering processes, such as those provided by electron-phonon coupling, are important.

The transition metal dipnictide $\mathrm{WP}_{2}$ is an ideal semimetal to study the role of coupling between the electron and phonon systems. The topological phase $\beta$ - $\mathrm{WP}_{2}$

*ks.burch@bc.edu

Published by the American Physical Society under the terms of the Creative Commons Attribution 4.0 International license. Further distribution of this work must maintain attribution to the author(s) and the published article's title, journal citation, and DOI. displays the largest magnetoresistance of any topological semimetal [3], with hints of hydrodynamic behavior at low temperatures [9]. In $\mathrm{WP}_{2}$, the resistivity decreases while the mobility increases by 4 and 5 orders of magnitude, respectively, between room temperature and $2 \mathrm{~K}$ [3]. Over the temperature range where the majority of this change occurs, the resistivity appears to be dominated by electron-phonon scattering $[3,10]$. Previous computational works also suggested that electron-phonon coupling plays a large role in determining the macroscopic transport properties of $\mathrm{WP}_{2}[11]$.

An important and related consideration is the phononelectron scattering, i.e., the scattering of phonons by electrons. Such scattering is of considerable importance when evaluating the lattice's effect on transport properties. For example, if phonon-electron scattering rates exceed phonon-phonon rates, momentum lost to phonons may be returned to the charge carriers, leading to an enhanced conductance [12-14]. Evidence for strong phonon-electron coupling in topological semimetals (TSM) has been primarily reported via optical spectroscopies that directly probe the system's phonons [15-18]. However, it is unclear what combination of factors contribute to the phononelectron scattering and thus what its role is in the transport behavior of TSMs. To this end, we perform a combined experimental, computational, and theoretical investigation of the phonon-electron coupling in $\mathrm{WP}_{2}$. Using Raman 
(a)

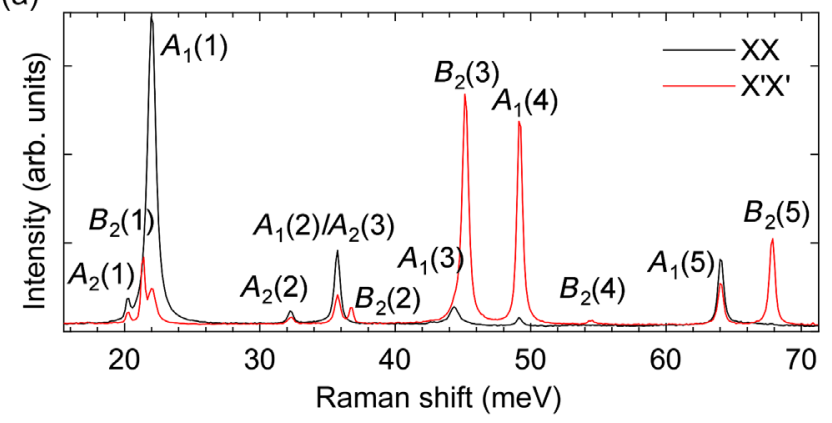

(b)

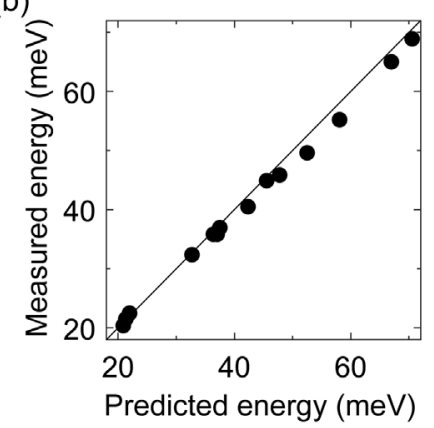

FIG. 1. (a) Room-temperature Raman spectra of an as-grown $\mathrm{WP}_{2}$ crystal surface in collinear $\mathrm{XX}$ and $\mathrm{X}^{\prime} \mathrm{X}^{\prime}$ polarization configurations. (b) Predicted phonon energies vs measured phonon energies. The solid line indicates ideal agreement.

spectroscopy, first-principles calculations, and symmetry analysis, we provide evidence that phonon-electron scattering dominates over phonon-phonon scattering over a wide temperature range. The ab initio calculations further elucidate the roles played by the relative phase space and phonon-electron coupling strength in the dominance of phonon-electron scattering.

\section{PHONON LINEWIDTH MEASUREMENTS}

We utilize Raman spectroscopy due to its sensitivity to symmetry, high-energy resolution, and the prevalence of phonon-electron coupling signatures in Raman spectra of other TSMs [15-18]. We begin by considering, in detail, the symmetry properties of $\mathrm{WP}_{2}$. The orthorhombic, nonsymmorphic space group $C m c 2_{1}$ leads to a total of 18 phonon modes. The 15 optical modes are all Raman active, and a group theoretical analysis [19] gives the irreducible representations $5 A_{1}, 3 A_{2}, 2 B_{1}$, and $5 B_{2}$. In Fig. 1(a), we show the room-temperature Raman spectra of an as-grown $\mathrm{WP}_{2}$ crystal surface measured in $\mathrm{XX}$ and $\mathrm{X}^{\prime} \mathrm{X}^{\prime}$ polarization configurations, revealing 13 of the 15 optical modes. Additional spectra from another $\mathrm{WP}_{2}$ crystal shown in the Supplemental Material [20] reveal one more mode $\left[B_{1}(2)\right]$, bringing the total number of observed modes to 14 of 15 . The full polarization dependence yields the mode assignments shown in Fig. 1(a), which are consistent with previous studies [21,22] (Table 1 in Ref. [20]). In Fig. 1(b), we show our measured versus our computationally predicted mode energies. The solid diagonal line indicates ideal agreement, and the reported values all fall within 3\% of this line.

We begin our search for signatures of phonon-electron coupling in the temperature dependence of the phonon energies. Typically, the phonon energy decreases as the temperature increases due to a combination of lattice expansion and anharmonic renormalization [23,24]. The temperature-dependent Raman spectra shown in Fig. 2(a) reveal behavior consistent with this result, with all observed modes shifting to lower energies as the temperature is increased from 10 to $300 \mathrm{~K}$. A previous report indicated an anomalous decrease in mode energies below about $25 \mathrm{~K}$ [22], which was interpreted as evidence for phononelectron coupling. In an initial run of low-temperature measurements, we observed similar behavior (see Supplemental Material [20]). However, in subsequent measurements, we found that this anomalous softening was removed by allowing more time for the $\mathrm{WP}_{2}$ crystals to come to thermal equilibrium with the cryostat at each temperature.

To extract the quantitative behavior of the phonon energies and linewidths, we fit each mode with a Voigt profile. While an asymmetric line shape can be an indication of phonon-electron coupling [16,25], we did not observe this in any $\mathrm{WP}_{2}$ mode. We also did not observe any electronic Raman scattering in the same spectral range as the optical phonons, which may explain the absence of any asymmetric line shapes, as a Fano line shape generally requires both a large coupling strength and large matrix element for electronic Raman scattering. In Fig. 2(b), we plot the percent change of each mode's energy and observe that, while most of the modes display changes below $1 \%$, the lowest-energy $A_{1}$ mode shifts by almost $2 \%$. While this could be a sign of phonon-electron coupling, the temperature dependence of phonon energies contains contributions from multiple sources, so unambiguous interpretation is challenging [24]. We therefore turn to the inspection of the phonon linewidths that only contain information related to the decay paths available to the phonon mode $[15,23]$.

The temperature-dependent linewidths of the two lowestenergy $A_{1}$ modes are plotted in Fig. 2(c). At temperatures below approximately $50 \mathrm{~K}$, the $A_{1}(1)$ mode's linewidth maintains a nearly constant value of $0.36 \mathrm{meV}$. Above this temperature, the linewidth grows rapidly, reaching a maximum value of approximately $0.65 \mathrm{meV}$ at approximately $200 \mathrm{~K}$, before slowly decreasing as it approaches room temperature. Though not as dramatic, a similar behavior is also seen in the $A_{1}(2)$ linewidth. This type of behavior is distinct from that predicted by optical phonon decay into acoustic phonons [26], as depicted in Fig. 2(e). In the Klemens model, an optical phonon of frequency $\omega$ is assumed to decay into two acoustic phonons of energy 
(a)

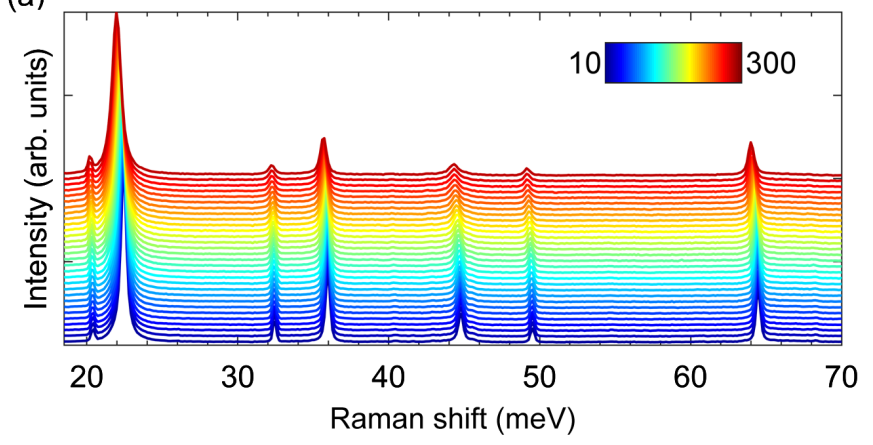

(c)

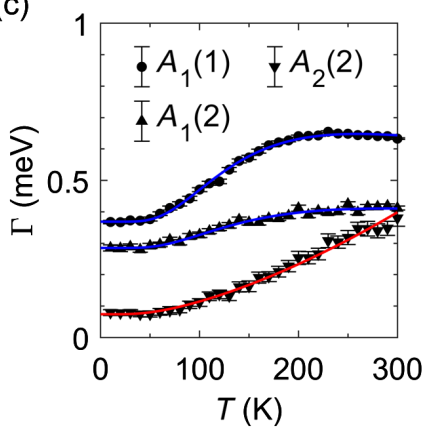

(d)

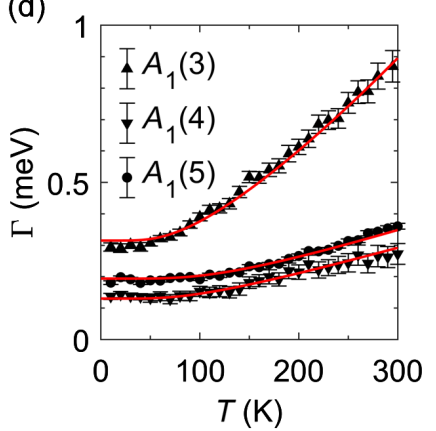

(b)

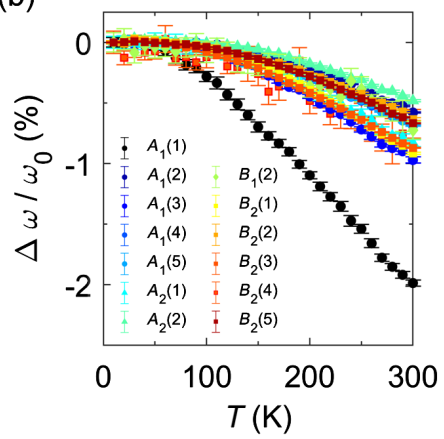

(e)

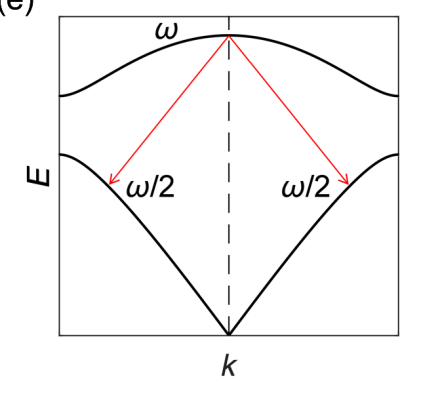

FIG. 2. (a) Temperature-dependent Raman spectra from 10 to $300 \mathrm{~K}$. Spectra are vertically offset. (b) Percent difference of the phonon energies from the $\omega_{0}$ value at $10 \mathrm{~K}$ as a function of temperature. The lowest-energy $A_{1}$ mode changes by twice the percent of any other mode. (c) Linewidth temperature dependence of the $A_{1}(1)$ and $A_{1}(2)$ modes, which have energies 22.4 and $35.8 \mathrm{meV}$ at $10 \mathrm{~K}$, respectively. The $A_{2}(2)$ mode at $32.4 \mathrm{meV}$ has an energy between that of the two $A_{1}$ modes. While both $A_{1}$ modes display an anomalous temperature dependence, the $A_{2}$ mode temperature dependence is that expected from anharmonic phonon decay. Fits in blue and red are those assuming decay into electron-hole pairs and acoustic phonons, respectively. (d) Three higher-energy $A_{1}$ modes, which also display the temperature dependence expected from anharmonic decay. (e) Schematic diagram showing the lowest-order anharmonic decay of an optical phonon with energy $\omega$ decay into two acoustic phonons with energy $\omega / 2$.

$\omega / 2$ and momentum $\pm \mathbf{k}$ to satisfy both energy and momentum conservation. The inclusion of higher-order processes in which an optical phonon decays into three acoustic phonons $(\omega / 3)$ constitutes the extended Klemens model $[23,24]$. Because of the bosonic nature of phonons, the temperature dependence in both of these models is governed by the Bose-Einstein distribution function $n_{B}(\omega, T)$. Therefore, the linewidth predicted by this model monotonically increases with temperature, a trend that is not displayed by the $A_{1}(1)$ or $A_{1}(2)$ modes. We instead identify their temperature dependence as resulting from decay into electron-hole pairs near the Fermi surface [15].

Linewidths indicative of optical phonon decay into electron-hole pairs have previously been observed in the semimetals TaAs [15,16], NbAs [15], $\mathrm{MoTe}_{2}$ [17], graphite [27,28], and $\mathrm{Cd}_{3} \mathrm{As}_{2}$ [18]. The model developed to describe such a behavior assumes that an optical phonon with $q=0$ decays into an electron-hole pair via an interband transition. The decay rate therefore depends on the difference in occupation functions between the electron and hole states [29]. Since it is assumed that the initial state is below $E_{F}$ and therefore occupied, the model developed for graphene predicts a linewidth monotonically decreasing as the temperature is raised due to the occupancy change of the filled and empty states, accounted for by Fermi functions $\left[n_{F}(\omega, T)\right]$. We modified this account for the nearly parallel spin-orbit split bands of $\mathrm{WP}_{2}: \quad \Gamma(T) \propto n_{F}\left(\omega_{a}, T\right)-$ $n_{F}\left(\omega_{a}+\omega_{\mathrm{ph}}, T\right)$, where $\omega_{\mathrm{ph}}$ is the energy of the optical phonon, and $\omega_{a}$ captures the distance between $E_{F}$ and the initial state of the electron [see Fig. 3(a), and additional details in the Supplemental Material [20] ]. The inclusion of the $\omega_{a}$ term can result in a nonmonotonic temperaturedependent behavior because, at low temperatures, the empty initial state prevents the transition. As the temperature increases, the initial state becomes thermally populated and the decay processes turn on. The solid blue lines in Fig. 2(c) are the results of fitting this model to our measured linewidths, and over the entire measured temperature range, it reproduces our experimental data very well. From the fits, we find $\omega_{a}$ values of $24.48 \pm 0.61 \mathrm{meV}$ and $22.64 \pm 1.73 \mathrm{meV}$ for the $A_{1}(1)$ and $A_{1}(2)$ modes, respectively, consistent with transitions close to the Fermi level. The relatively close energies of the two $A_{1}$ modes are likely responsible for the similarity in $\omega_{a}$ values obtained for each mode.

Having identified the mechanism responsible for the anomalous behavior in the two lowest-energy $A_{1}$ modes, we turn to the $A_{2}(2)$ mode whose energy is between the 
(a)

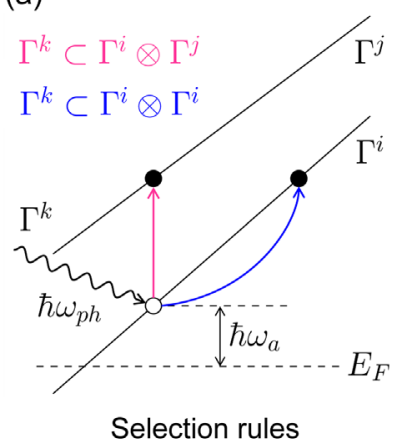

(b)

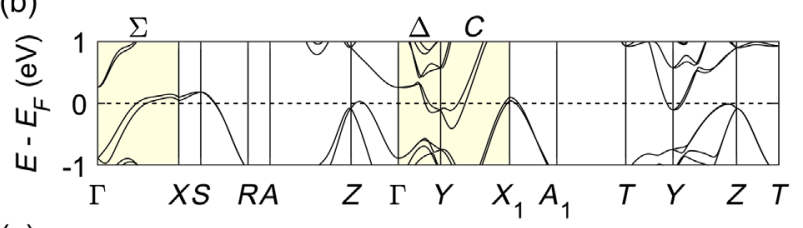

(c)

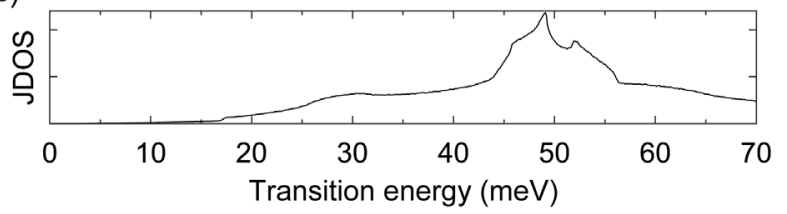

FIG. 3. (a) Schematic depicting interband (pink arrow) and intraband (blue arrow) transitions by optical phonons. An energy $\hbar \omega_{a}$ separates the initial electron state from the Fermi energy $E_{F}$. The electronic bands are labeled by irreducible representations $\Gamma^{i}$ and $\Gamma^{j}$, while the optical phonon of energy $\hbar \omega_{\mathrm{ph}}$ has the representation $\Gamma^{k}$. (b) Calculated electronic band structure of $\mathrm{WP}_{2}$. The shaded regions indicate symmetry lines where scattering by $\Gamma$-point optical phonons is allowed and energetically possible. (c) Weighted joint density of states for vertical $(q=0)$ transitions between states within $\pm 70 \mathrm{meV}$ of $E_{F}$.

$A_{1}(1)$ and $A_{1}(2)$ modes. As can be seen in Fig. 2(c), the linewidth of this mode does not display any anomalous behavior and instead follows the dependence expected from the model of anharmonic decay. A fit to the data using the extended Klemens model, plotted as a solid red line, captures the observed trend and confirms this assessment. This observation suggests that the symmetry of the phonon mode may play a role in determining whether it decays into electron-hole pairs or other phonons, which is further supported by the lack of anomalous behavior in the $B_{1}$ or $B_{2}$ modes (see Supplemental Material [20]). However, symmetry is unlikely to be the only determining factor, as evidenced by the linewidths for the three higher-energy $A_{1}$ modes plotted in Fig. 2(d). Despite sharing the same symmetry, these three modes display behavior consistent with the anharmonic model of decay. Taken together, these observations indicate that both the symmetry and energy of the optical phonon are factors in determining its primary decay channels. As discussed later, the availability of phonon decay paths, the phonon-electron coupling strength, and momentum conservation also contribute.

\section{PHONON DECAY PATHS}

\section{A. Energy conservation}

To understand the role of phonon energy, we turn to the available phase space for decay into electron-hole pairs. In Fig. 3(b), we show the calculated electronic band structure for $\mathrm{WP}_{2}$ within $\pm 1 \mathrm{eV}$ of the Fermi level, which agrees with previous work [11,30]. Along the $\Sigma(\Gamma \rightarrow \mathrm{X})$, $\Delta(\Gamma \rightarrow \mathrm{Y})$, and $\mathrm{C}\left(\mathrm{Y} \rightarrow \mathrm{X}_{1}\right)$ cuts, the gaps between neighboring bands appear to be on an energy scale similar to the optical phonons. To confirm this, Fig. 3(c) shows a low-temperature weighted joint density of states (JDOS) (see the Appendix) for vertical $(q=0)$ transitions between states within an optical phonon energy $( \pm 70 \mathrm{meV})$ of $E_{F}$. Available transitions exist across the entire range, with the largest JDOS occurring between 40 and $60 \mathrm{meV}$. The $A_{1}$ modes that display phononelectron dominated linewidths have energies of 22.4 and $35.8 \mathrm{meV}$, which have a comparatively smaller electronic JDOS. By contrast, the $A_{1}$ modes that fall in the 40-60 meV range display linewidths governed by anharmonic decay, indicating that the availability of electronic states is not the largest contributing factor to the phonon linewidths. As discussed later, we find that momentum conservation is also required to understand the difference between these $A_{1}$ modes.

\section{B. Selection rules}

Before addressing momentum conservation, it is instructive to consider the phonon symmetry via the selection rules for transitions between electronic bands. Because of lattice symmetries, there are only certain symmetry points and lines that will allow transitions by $q \approx 0$ phonons to occur. The $\Sigma, \Delta$, and $\mathrm{C}$ cuts, which allow these transitions and intersect the Fermi surface, are shaded in Fig. 3(b). In addition to these cuts, there are other parts of the Fermi surface that exist at general $k$-points, not shown in Fig. 3(b), for which $q \approx 0$ transitions are allowed. The irreducible representations of the electronic bands along the highsymmetry cuts may be found from compatibility relations $[31,32]$ as summarized in Table I. With the inclusion of

TABLE I. Compatibility relations along the highlighted cuts and adjacent points in Fig. 3(b).

\begin{tabular}{lcccc}
\hline \hline$\Sigma$ & $\Gamma$ & $\Delta$ & $\mathrm{Y}$ & $\mathrm{C}$ \\
\hline$\Sigma^{(1)}$ & $\Gamma^{(1)}\left(A_{1}\right)$ & $\Delta^{(1)}$ & $\mathrm{Y}^{(1)}$ & $\mathrm{C}^{(2)}$ \\
$\Sigma^{(2)}$ & $\Gamma^{(2)}\left(A_{2}\right)$ & $\Delta^{(2)}$ & $\mathrm{Y}^{(2)}$ & $\mathrm{C}^{(1)}$ \\
$\Sigma^{(2)}$ & $\Gamma^{(3)}\left(B_{2}\right)$ & $\Delta^{(1)}$ & $\mathrm{Y}^{(3)}$ & $\mathrm{C}^{(1)}$ \\
$\Sigma^{(1)}$ & $\Gamma^{(4)}\left(B_{1}\right)$ & $\Delta^{(2)}$ & $\mathrm{Y}^{(4)}$ & $\mathrm{C}^{(2)}$ \\
$\bar{\Sigma}^{(3)} \oplus \bar{\Sigma}^{(4)}$ & $\bar{\Gamma}^{(5)}$ & $\bar{\Delta}^{(3)} \oplus \bar{\Delta}^{(4)}$ & $\overline{\mathrm{Y}}^{(5)}$ & $\overline{\mathrm{C}}^{(3)} \oplus \overline{\mathrm{C}}^{(4)}$ \\
\hline \hline
\end{tabular}



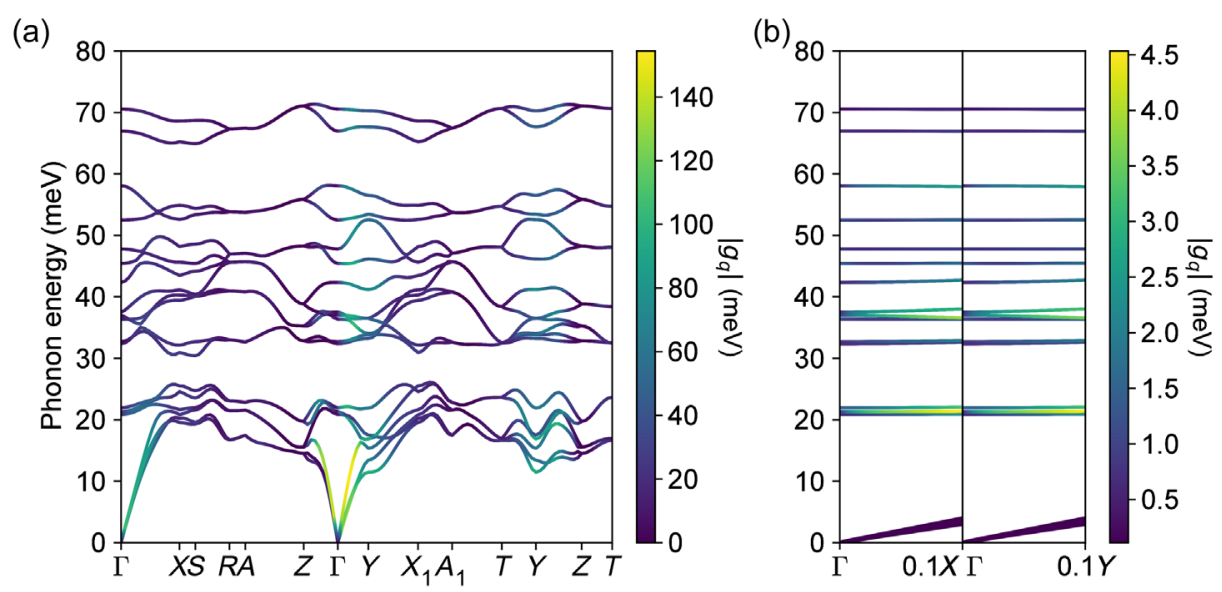

FIG. 4. (a) Calculated phonon dispersion of $\mathrm{WP}_{2}$. The effective normalized phonon-electron matrix element is projected onto each phonon mode $(q, \omega)$. The acoustic phonon modes have much stronger coupling than the optical modes. However, when comparing different optical modes, the lower-energy modes $A_{1}(1)$ and $A_{1}(2)$ show a significant enhancement of phonon-electron coupling compared to that of the higher-energy ones. (b) Close-up view of the phonon-electron coupling strength for small $q$ near $\Gamma$, highlighting the largely enhanced coupling strength at finite $q$. Only the optical modes are colored according to coupling for clarification.

spin-orbit coupling, all of the electronic bands belong to representations from the double space group, indicated by a bar.

The selection rules for intraband and interband transitions are found by taking the direct product of the irreducible representations for the electronic bands which, in general, produces a reducible representation and decomposing it into its irreducible components. A phononinduced transition between these bands is symmetry allowed only for phonons that transform as irreducible representations contained in this decomposition. This case is schematically depicted in Fig. 3(a), where interband transitions are depicted by a pink arrow and representations, while intraband transitions are shown with a blue arrow and representations. We note that while the only interband transition shown has $q=0, q \neq 0$ transitions are also possible and will, in general, have different selection rules than those with $q=0$. The full details concerning the decomposition of the direct products can be found in the Supplemental Material [20]; here, we summarize the main results. If we restrict the phonon decay via electronic interband and intraband transitions to points along the symmetry lines shown in Fig. 3(b), we find that phonons of $\Gamma^{(2)}, \Gamma^{(3)}$, or $\Gamma^{(4)}\left(\mathrm{A}_{2}, \mathrm{~B}_{2}\right.$, or $\mathrm{B}_{1}$, respectively) symmetry can produce interband transitions, while intraband transitions can result from $\Gamma^{(1)}\left(A_{1}\right), \Gamma^{(3)}$, and $\Gamma^{(4)}$ phonons. However, when we consider transitions between general $k$ points or for points close to the lines of symmetry, we find that (a) there is no longer any distinction between the selection rules for interband or intraband transitions and (b) phonons of any representation may produce both types of transitions. Since the majority of the Fermi surface exists at general $k$-points [33], we conclude that both interband and intraband processes likely contribute to the observed linewidth behavior for the $A_{1}(1)$ and $A_{1}(2)$ modes.
However, we note that, per these selection rules, only interband transitions with $q \neq 0$ are possible for the $A_{1}$ phonons. We therefore consider whether momentum conservation can be satisfied under our experimental conditions.

\section{Momentum conservation}

For interband phonon decay into electron-hole pairs, the momentum and energy conservation is trivially satisfied. As shown in Fig. 3(c), interband transitions with $q=0$ exist across the entire range of optical phonon energies. Interband transitions with $q \approx 0$ will therefore have a very similar phase space. For intraband transitions, we explicitly estimate both the momentum provided by the photons and the momentum required for the electronic transition. Near the Fermi energy, we assume the electronic bands are approximately linear [34], with a slope given by the experimentally measured Fermi velocities [9]. The momentum obtained thus depends on the specific electron or hole pocket under consideration (see Supplemental Material [20] for details of the calculation), but for the $A_{1}(1)$ and $A_{1}(2)$ phonons, we find $q \approx 8.57 \times 10^{7} \mathrm{~m}^{-1}$ and $q \approx 1.37 \times 10^{8} \mathrm{~m}^{-1}$, respectively. In the backscattering configuration, the transferred momentum is found from the relation $q \leq 4 \pi n_{i}(\lambda) / \lambda$, where $n_{i}(\lambda)$ is the wavelengthdependent index of refraction for the $i$ th crystal direction and $\lambda$ is the wavelength of the optical excitation. We use the calculated index of refraction (see Supplemental Material [20]) and find $q \leq 9.68 \times 10^{7} \mathrm{~m}^{-1}$. We therefore see that the momentum provided by the laser is enough to produce $A_{1}(1)$ phonons capable of intraband transitions. However, it falls short for the $A_{1}(2)$ phonon, which suggests that while both interband and intraband processes may be available for the $A_{1}(1)$ phonon, only interband processes are available for the $A_{1}(2)$ phonon. The lower availability of decay paths for 
the $A_{1}(2)$ mode may thus help explain why, despite having similar coupling strengths, the linewidth of the $A_{1}(1)$ mode appears to be more strongly influenced by the phononelectron decay processes. Regardless, it is clear that the momentum necessary for an intraband transition by the higher-energy $A_{1}$ modes, which we estimate would have a minimum value of $q \approx 1.71 \times 10^{8} \mathrm{~m}^{-1}$, cannot be provided by our laser, and thus only interband transitions may contribute to the phonon-electron component of their linewidths.

\section{Phonon-electron coupling}

The presence of $A_{2}, B_{1}$, and $B_{2}$ symmetries in the selection rules, combined with the lack of anomalous behavior in the linewidths for phonons of these symmetries (see Supplemental Material [20]), suggests that the phonon-electron coupling strength needs to be considered. Figure 4(a) presents the calculated phonon dispersion for $\mathrm{WP}_{2}$, with the phonon-electron matrix element shown on each mode. Overall, the acoustic branches display greater coupling, while the optical modes have considerable coupling along the $\Delta$ and $\mathrm{T}$ to $\mathrm{Y}$ to $\mathrm{Z}$ cuts, corresponding to phonon wave vectors that connect separate parts of the Fermi surface [15]. In Fig. 4(b), we show a close-up of the optical phonon dispersion along the $\Sigma$ and $\Delta$ cuts (for mode-resolved plots of these same data, see Supplemental Material [20]). For $q=0$, the phonon-electron coupling strength for all modes is very weak, with most modes displaying a $|g|$ (see Appendix) on the order of $0.1 \mathrm{meV}$. This comparatively weak coupling suggests that interband transitions with $q=0$ do not represent a significant source of phonon-electron scattering. For finite $q$, we see that for the $A_{1}(1)$ and $A_{1}(2)$ modes, the coupling strength increases by an order of magnitude, while for the higher-energy modes, the increase in coupling strength is not as dramatic. Despite showing similar increases in coupling strength, the $A_{2}(1)$ and $B_{2}(1)$ modes do not display a clear phononelectron contribution to their linewidths, which suggests that for modes of these symmetries, the connection between higher phonon-electron coupling and linewidth behavior is less clear. We explicitly confirm this result by calculating the phonon-electron linewidth contribution at ambient temperatures. In the Supplemental Material [20], we show that the $A_{1}(1)$ and $A_{1}(2)$ modes possess significantly larger phonon-electron linewidth contributions compared to the other phonon modes. Overall, we find these trends in the coupling strength to be consistent with our measurements and interpretation of the phonon linewidths.

\section{DISCUSSION}

The calculated phonon dispersions may also explain why phonon-electron decay dominates over phonon-phonon decay. As shown in Fig. 4(a), there is no energy separation between the acoustic branches and the lowest-energy optical modes. These lower-energy optical modes involve large displacements of the heavy $\mathrm{W}$ atoms compared to the higher-energy modes, which predominantly feature displacement of the lighter $\mathrm{P}$ atoms (see Supplemental Material [20]). The heavy $\mathrm{W}$ atoms also lead to the very narrow bandwidth of the acoustic branches, similar to the "acoustic bunching" discussed for high thermal conductivity materials [35]. These features suggest there is a restricted phase space for decay into acoustic phonons, leading to longer phonon-phonon lifetimes for low-energy modes, allowing phonon-electron processes to dominate the optical-mode decay behavior. For the higher-energy modes, the available phonon-phonon phase space is much larger (Supplemental Material [20]), explaining the dominance of phonon-phonon processes for the higher-energy $A_{1}$ modes.

The bunching arguments presented above also apply to the phonon-phonon scattering rates for the acoustic modes [35]. Given the much larger phonon-electron coupling strengths of the acoustic modes [Fig. 4(a)], we expect that this could lead to a predominance of phonon-electron scattering for these modes. We note that similar conclusions have been drawn from previous computational works as well [11], yet they did not explicitly estimate the relevant phonon lifetimes. In the Supplemental Material [20], we show calculations of the phonon-phonon contribution to phonon lifetimes, $\tau_{\text {ph-ph }}$, at high and low temperatures, as well as the phonon-electron lifetime contribution, $\tau_{\mathrm{ph}-\mathrm{e}}$. At room temperature $(300 \mathrm{~K})$, the median $\tau_{\text {ph-ph }}$ for acoustic modes with energy less than about $10 \mathrm{meV}$ is about $25 \mathrm{ps}$, while at low temperature $(50 \mathrm{~K})$, it increases to about $10^{3}$ ps. By comparison, the phonon-electron lifetimes for the same modes are estimated to have a median value of about $10 \mathrm{ps}$. At low temperatures, we therefore find a ratio $\tau_{\text {ph-e }} / \tau_{\text {ph-ph }} \approx 10^{-1}$, the hierarchy of which strongly suggests that phonon-electron scattering is the dominant scattering pathway for these low-energy acoustic phonons. If such phonon-electron processes are indeed the dominant source of scattering over phonon-phonon scattering, momentum provided to the phonons by electron-phonon scattering, which might otherwise be lost, may be returned to the electrons and lead to an enhanced conductivity. We note that these arguments are similar to those made for understanding the phonon drag effect [12], yet further studies are needed to confirm its existence and its influence on the overall transport properties.

Finally, we comment on the role that the topological features of $\beta-\mathrm{WP}_{2}$ 's electronic structure may play in the phonon-electron decay mechanism we report on. In $\beta-\mathrm{WP}_{2}$, the Weyl nodes are located hundreds of meV below the Fermi surface [30,34]. The impact of these nodes on the transport properties of bulk $\mathrm{WP}_{2}$, which are typically sensitive to the Fermi surface, is therefore questionable. For the particular phonon-electron decay mechanism discussed here, the nodes themselves do not seem to play any role in facilitating the effect since the optical phonon 
energies are an order of magnitude smaller than the depth of the Weyl nodes. We do note, though, that the spin-orbit coupling giving rise to the Weyl nodes also produces the band splitting that enables interband phonon decay paths [30]. Further evidence that this effect is not related to the topological nature of $\beta$ - $\mathrm{WP}_{2}$ may be found in another recent study of the phonon dynamics of $\alpha-\mathrm{WP}_{2}$, which displayed nearly identical linewidth behavior, despite $\alpha$ - $\mathrm{WP}_{2}$ being topologically trivial [22]. While the topological nature of the material may not play a direct role in the phonon-electron behavior discussed here, the features of electronic bands in topological materials may provide the proper conditions for such behavior to occur. If crossed and linearly dispersing bands are found near the Fermi energy, then interband optical phonon decay may occur for phonons of arbitrary energy. As noted earlier, similar linewidth behavior has indeed been reported for TaAs and NbAs [15,16], two prototypical Weyl semimetals with Weyl nodes near their Fermi surfaces. In addition, the heavy atoms with large spin-orbit coupling found in many topological materials also lead to a reduction in the bandwidth of the acoustic phonons, limiting the available phase space for phonon-phonon scattering as discussed above. We thus conclude that while the effects we observed here could be possible in a semimetallic system regardless of its topological nature, the unique features of topological semimetals may provide a canvas well suited for their expression.

\section{CONCLUSIONS}

By examining the temperature dependence of the optical phonon linewidths of $\mathrm{WP}_{2}$, we identified electron-hole decay as the primary path for the lowest-energy $A_{1}$ optical phonon modes over a broad temperature range. Through the use of group theory, we further identified that both interband and intraband processes with finite momentum may contribute to this behavior. Combined with firstprinciples calculations, we also explained the importance of the phonon-electron coupling strength and the relative availability of phonon-phonon decay paths. This improved understanding of the phonon-induced intraband and interband scattering is valuable in a material where electronphonon coupling has been suggested to play a vital role in its macroscopic transport properties. We anticipate that these results could be beneficial to understanding similar phonon behavior that has recently been observed in other Weyl semimetals [15,17]. In addition, the much larger phonon-electron coupling strengths calculated for the acoustic modes suggest they may display similar behavior and primarily scatter off of charge carriers at low temperatures, potentially leading to enhancements of the conductivity [12]. Confirmation of such behavior will require future investigations with probes capable of accessing the acoustic modes such as, e.g., resonant inelastic x-ray spectroscopy, neutron scattering, or Brillouin scattering.
Overall, we note that while the results we present here support the conclusion that phonons play a significant role in the transport properties of $\mathrm{WP}_{2}$, more work will be required to understand the more general role of phononelectron coupling in the dynamics of topological semimetals at large.

\section{ACKNOWLEDGMENTS}

Analysis and measurements performed by G. B. O. and V. M. P. as well as work done by K. S. B. were supported by the U.S. Department of Energy (DOE), Office of Science, Office of Basic Energy Sciences under Award No. DE-SC0018675. Y. W. is supported by the STC Center for Integrated Quantum Materials, NSF Grant No. DMR1231319. Calculations by Y.W. and C.A.C.G. used resources of the National Energy Research Scientific Computing Center, a DOE Office of Science User Facility supported by the Office of Science of the U.S. Department of Energy under Contract No. DE-AC0205CH11231, as well as resources at the Research Computing Group at Harvard University. Additional calculations were performed using resources from the Department of Defense High Performance Computing Modernization Program through the Army Research Office MURI Grant on Ab Initio Solid-State Quantum Materials: Design, Production, and Characterization at the Atomic Scale (Grant No. 18057522). C. A. C. G. was supported by the NSF Graduate Research Fellowship Program under Grant No. DGE1745303. P. N. gratefully acknowledges support through Grant No. GBMF8048 from the Gordon and Betty Moore Foundation. J. G. and C. F. acknowledge financial support by the Deutsche Forschungsgemeinschaft (DFG, German Research Foundation) under Germany's Excellence StrategyEXC2147 “ct.qmat" (Project-ID No. 390858490).

G. B. O. and V. M. P. performed the primary measurements, analyzed the data, and provided the plots. G. B. O. performed the group theory analysis. Y.W., C. A.C. G., and P. N. performed the first-principles calculations. J. G. and C.F. prepared the single crystal samples. G. B. O. and K. S. B. wrote the manuscript, with contributions from other authors. P. N. and K. S. B. jointly conceived the project and ideas presented.

\section{APPENDIX: METHODS}

\section{Experimental details}

Raman spectra were collected in the backscattering configuration using a custom-built setup [36]. The 532nm light from a frequency-doubled Nd:YAG laser was focused by a $100 \mathrm{X}$ long-working distance objective to a spot size of approximately $2 \mu \mathrm{m}$ in a Montana Instruments cryostation, which enabled access to temperatures from 300 to $10 \mathrm{~K}$. An incident power of less than $250 \mu \mathrm{W}$ was 
used to achieve satisfactory signal-to-noise ratios, with minimal laser-induced heating, which was checked using the Stokes to anti-Stokes ratio at all temperatures where the anti-Stokes signal was measurable ( $\gtrsim 100 \mathrm{~K})$. Polarization dependence was performed by the rotation of a double Fresnel rhomb, which acts as a broadband half-waveplate (see Supplemental Material [20]). Any spectra presented have been averaged, their background dark counts have been subtracted, and cosmic rays have been removed from them [37].

Fitting of the phonon features in our data was accomplished by a Levenburg-Marquardt least-squares fitting algorithm. Phonons were fit using a Voigt profile, whereby a Lorentzian representing the intrinsic phonon response is convoluted with a Gaussian to account for the instrument-induced broadening. A Gaussian linewidth of $0.217 \mathrm{meV}\left(1.75 \mathrm{~cm}^{-1}\right)$, determined from fitting of the Rayleigh line, was used for the fitting. The average energy resolution of our system was $0.137 \mathrm{meV}\left(1.1 \mathrm{~cm}^{-1}\right)$. Changes in phonon energies or linewidths smaller than the resolution of our system were able to be resolved, provided that the linewidth of the mode was broad enough such that the spectral feature was spread over many individual pixels.

\section{Computational details}

Calculations were performed using the $a b$ initio package QUANTUM ESPRESSO [38,39] to evaluate electronic band structure, phonon dispersion, and electronphonon interactions, combining density functional theory (DFT) and density functional perturbation theory (DFPT). Fully relativistic optimized norm-conserving Vanderbilt (ONCV) pseudopotentials [40] for the PBE exchange-correlation functional [41] were used for both $\mathrm{W}$ and $\mathrm{P}$, which allowed us to calculate the electronic structure, including SOC. Furthermore, we calculated electron-phonon coupling matrix elements of $\mathrm{WP}_{2}$ with the electron-phonon Wannier package [42], interpolating the maximally localized Wannier functions $[43,44]$ on initial coarse $8 \times 8 \times 8 k$ and $4 \times 4 \times 4 q$ grids, from which we Fourier transformed back to much finer $60 \times$ $60 \times 80 k$ and $q$ grids.

The effective phonon-electron coupling strength for a phonon with wave vector $\mathbf{q}$ and branch index $\nu$ is defined as $\left|g_{\mathbf{q}, \nu}\right|=\sqrt{\lambda_{\mathbf{q}, \nu}}$, where

$\lambda_{\mathbf{q}, \nu}=\frac{1}{N_{F} \omega_{\mathbf{q}, \nu}} \sum_{m n, \mathbf{k}} w_{k}\left|g_{m n, \nu}(\mathbf{k}, \mathbf{q})\right|^{2} \delta\left(\varepsilon_{n \mathbf{k}}\right) \delta\left(\varepsilon_{m \mathbf{k}+\mathbf{q}}\right)$.

Here, $\omega_{\mathbf{q}, \nu}$ is the phonon frequency, $w_{k}$ is the weight of the k-points, and $N_{F}$ is the density of states at the Fermi energy. Note that $\varepsilon_{n \mathbf{k}}$ and $\varepsilon_{m \mathbf{k}+\mathbf{q}}$ are the electron band energies with wave vector $\mathbf{k}(\mathbf{k}+\mathbf{q})$ and band index $n(m)$. The electron-phonon matrix element is given by

$$
g_{m n, \nu}(\mathbf{k}, \mathbf{q})=\left\langle\psi_{m \mathbf{k}+\mathbf{q}}\left|\partial_{\mathbf{q} \nu} V\right| \psi_{n \mathbf{k}}\right\rangle
$$

with $\psi_{n \mathbf{k}}$ being the electronic wave function and $\partial_{\mathbf{q} \nu} V$ the derivative of the self-consistent potential associated with a phonon $\omega_{\mathbf{q} \nu}$.

For the weighted JDOS and refractive index evaluations, separate DFT calculations were carried out using the implementation in Joint Density Functional Theory software JDFTx [45]. Fully relativistic ultrasoft pseudopotentials $[46,47]$ for the PBEsol exchange-correlation functional [48] were used, as well as a uniform $6 \times 6 \times 8 k$ grid for the 6-atom standard primitive unit cell, an energy cutoff of 28 Hartrees, Fermi-Dirac smearing with a 0.01-Hartree width, and a $3 \times 3 \times 2$ phonon supercell. Maximally localized Wannier functions $[43,44]$ were similarly obtained to interpolate quantities for Monte Carlo Brillouin zone integration on finer $k$ and $q$ meshes [49]. Each transition contributing to the JDOS at that transition energy is weighted by the factor $n_{F}\left(E_{\mathbf{k}, n}, T\right)-$ $n_{F}\left(E_{\mathbf{k}, m}, T\right)$, where $n_{F}(E, T)$ is the Fermi-Dirac distribution function, and the transition occurs between energies $E_{\mathbf{k}, n}$ and $E_{\mathbf{k}, m}$ at the momentum $\mathbf{k}$ for two bands indexed by $n$ and $m$. The results presented in Fig. 3(c) are for $T=4 \mathrm{~K}$, with transitions restricted to be between states within $\pm 70 \mathrm{meV}$ of the Fermi level. The refractive index calculations are discussed in the Supplemental Material [20].

[1] C. Shekhar, A. K. Nayak, Y. Sun, M. Schmidt, M. Nicklas, I. Leermakers, U. Zeitler, Y. Skourski, J. Wosnitza, Z. Liu, Y. Chen, W. Schnelle, H. Borrmann, Y. Grin, C. Felser, and B. Yan, Extremely Large Magnetoresistance and Ultrahigh Mobility in the Topological Weyl Semimetal Candidate NbP, Nat. Phys. 11, 645 (2015).

[2] Z. Wang, Y. Zheng, Z. Shen, Y. Lu, H. Fang, F. Sheng, Y. Zhou, X. Yang, Y. Li, C. Feng, and Z.-A. Xu, HelicityProtected Ultrahigh Mobility Weyl Fermions in NbP, Phys. Rev. B 93, 121112(R) (2016).

[3] N. Kumar, Y. Sun, N. Xu, K. Manna, M. Yao, V. Süß, I. Leermakers, O. Young, T. Förster, M. Schmidt, H. Borrmann, B. Yan, U. Zeitler, M. Shi, C. Felser, and C. Shekhar, Extremely High Magnetoresistance and Conductivity in the Type-II Weyl Semimetals $\mathrm{WP}_{2}$ and $\mathrm{MoP}_{2}$, Nat. Commun. 8, 1642 (2017).

[4] M. N. Ali, J. Xiong, S. Flynn, J. Tao, Q. D. Gibson, L. M. Schoop, T. Liang, N. Haldolaarachchige, M. Hirschberger, N. P. Ong, and R. J. Cava, Large, Non-saturating Magnetoresistance in $\mathrm{WTe}_{2}$, Nature (London) 514, 205 (2014).

[5] P. Narang, C. A. C. Garcia, and C. Felser, The Topology of Electronic Band Structures, Nat. Mater. (2020) https:// doi.org/10.1038/s41563-020-00820-4.

[6] H.-Y. Yang, T. Nummy, H. Li, S. Jaszewski, M. Abramchuk, D. S. Dessau, and F. Tafti, Extreme Magnetoresistance in 
the Topologically Trivial Lanthanum Monopnictide LaAs, Phys. Rev. B 96, 235128 (2017).

[7] E. Mun, H. Ko, G. J. Miller, G. D. Samolyuk, S. L. Bud'ko, and P.C. Canfield, Magnetic Field Effects on Transport Properties of $\mathrm{PtSn}_{4}$, Phys. Rev. B 85, 035135 (2012).

[8] J. Du, Z. Lou, S. N. Zhang, Y. Zhou, B. Xu, Q. Chen, Y. Tang, S. Chen, H. Chen, Q. Zhu, H. Wang, J. Yang, Q. S. Wu, O. V. Yazyev, and M. Fang, Extremely Large Magnetoresistance in the Topologically Trivial Semimetal $\alpha$-WP 2 , Phys. Rev. B 97, 245101 (2018).

[9] J. Gooth, F. Menges, N. Kumar, V. Sü $\beta$, C. Shekhar, Y. Sun, U. Drechsler, R. Zierold, C. Felser, and B. Gotsmann, Thermal and Electrical Signatures of a Hydrodynamic Electron Fluid in Tungsten Diphosphide, Nat. Commun. 9, 4093 (2018).

[10] A. Jaoui, B. Fauqué, C. W. Rischau, A. Subedi, C. Fu, J. Gooth, N. Kumar, V. Süß, D. L. Maslov, C. Felser, and K. Behnia, Departure from the Wiedemann-Franz Law in $\mathrm{WP}_{2}$ Driven by Mismatch in T-Square Resistivity Prefactors, npj Quantum Mater. 3, 64 (2018).

[11] J. Coulter, R. Sundararaman, and P. Narang, Microscopic Origins of Hydrodynamic Transport in the Type-II Weyl Semimetal $\mathrm{WP}_{2}$, Phys. Rev. B 98, 115130(R) (2018).

[12] R. Peierls, Zur Frage des Elektrischen Widerstandsgesetzes für Tiefe Temperaturen, Ann. Phys. (Leipzig) 404, 154 (1932).

[13] N. Wiser, The Electrical Resistivity of the Simple Metals, Contemp. Phys. 25, 211 (1984).

[14] G. Varnavides, A. S. Jermyn, P. Anikeeva, C. Felser, and P. Narang, Electron hydrodynamics in anisotropic materials, Nat. Commun. 11, 4710 (2020).

[15] J. Coulter, G. B. Osterhoudt, C. A. C. Garcia, Y. Wang, V. M. Plisson, B. Shen, N. Ni, K. S. Burch, and P. Narang, Uncovering Electron-Phonon Scattering and Phonon Dynamics in Type-I Weyl Semimetals, Phys. Rev. B 100, 220301(R) (2019).

[16] B. Xu, Y. M. Dai, L. X. Zhao, K. Wang, R. Yang, W. Zhang, J. Y. Liu, H. Xiao, G. F. Chen, S. A. Trugman, J.-X. Zhu, A. J. Taylor, D. A. Yarotski, R. P. Prasankumar, and X. G. Qiu, Temperature-Tunable Fano Resonance Induced by Strong Coupling between Weyl Fermions and Phonons in TaAs, Nat. Commun. 8, 14933 (2017).

[17] A. Zhang, X. Ma, C. Liu, R. Lou, Y. Wang, Q. Yu, Y. Wang, T.-1. Xia, S. Wang, L. Zhang, X. Wang, C. Chen, and Q. Zhang, Topological Phase Transition between Distinct Weyl Semimetal States in $\mathrm{MoTe}_{2}$, Phys. Rev. B $\mathbf{1 0 0}$, 201107(R) (2019).

[18] A. Sharafeev, V. Gnezdilov, R. Sankar, F. C. Chou, and P. Lemmens, Optical Phonon Dynamics and Electronic Fluctuations in the Dirac Semimetal $\mathrm{Cd}_{3} \mathrm{As}_{2}$, Phys. Rev. B 95, 235148 (2017).

[19] E. Kroumova, M. Aroyo, J. Perez-Mato, A. Kirov, C. Capillas, S. Ivantchev, and H. Wondratschek, Bilbao Crystallographic Server: Useful Databases and Tools for PhaseTransition Studies, Phase Transit. 76, 155 (2003).

[20] See Supplemental Material at http://link.aps.org/ supplemental/10.1103/PhysRevX.11.011017 for additional data for all phonon modes, extended discussion of the phonon lifetimes, details about the direct product decom- positions for the symmetry analysis, and additional computational results.

[21] B. Su, Y. Song, Y. Hou, X. Chen, J. Zhao, Y. Ma, Y. Yang, J. Guo, J. Luo, and Z.-G. Chen, Strong and Tunable Electrical Anisotropy in Type-II Weyl Semimetal Candidate $\mathrm{WP}_{2}$ with Broken Inversion Symmetry, Adv. Mater. 31, 1903498 (2019).

[22] D. Wulferding, P. Lemmens, F. Büscher, D. Schmeltzer, C. Felser, and C. Shekhar, Effect of Topology on Quasiparticle Interactions in the Weyl Semimetal $\mathrm{WP}_{2}$, Phys. Rev. B 102, 075116 (2020).

[23] M. Balkanski, R. F. Wallis, and E. Haro, Anharmonic Effects in Light Scattering Due to Optical Phonons in Silicon, Phys. Rev. B 28, 1928 (1983).

[24] Y. Tian, S. Jia, R. J. Cava, R. Zhong, J. Schneeloch, G. Gu, and K.S. Burch, Understanding the Evolution of Anomalous Anharmonicity in $\mathrm{Bi}_{2} \mathrm{Te}_{3-x} \mathrm{Se}_{x}$, Phys. Rev. B 95, 094104 (2017).

[25] U. Fano, Effects of Configuration Interaction on Intensities and Phase Shifts, Phys. Rev. 124, 1866 (1961).

[26] P. G. Klemens, Anharmonic Decay of Optical Phonons, Phys. Rev. 148, 845 (1966).

[27] P. Giura, N. Bonini, G. Creff, J. B. Brubach, P. Roy, and M. Lazzeri, Temperature Evolution of Infrared-and RamanActive Phonons in Graphite, Phys. Rev. B 86, 121404(R) (2012).

[28] H.-N. Liu, X. Cong, M.-L. Lin, and P.-H. Tan, The Intrinsic Temperature-Dependent Raman Spectra of Graphite in the Temperature Range from $4 \mathrm{~K}$ to $1000 \mathrm{~K}$, Carbon 152, 451 (2019).

[29] M. Lazzeri, S. Piscanec, F. Mauri, A. C. Ferrari, and J. Robertson, Phonon Linewidths and Electron-Phonon Coupling in Graphite and Nanotubes, Phys. Rev. B 73, 155426 (2006).

[30] G. Autès, D. Gresch, M. Troyer, A. A. Soluyanov, and O. V. Yazyev, Robust Type-II Weyl Semimetal Phase in Transition Metal Diphosphides $X \mathrm{P}_{2}(X=\mathrm{Mo}, W)$, Phys. Rev. Lett. 117, 066402 (2016).

[31] J. L. Birman, Space Group Selection Rules: Diamond and Zinc Blende, Phys. Rev. 127, 1093 (1962).

[32] L.-C. Chen, R. Berenson, and J. L. Birman, Space-Group Selection Rules: "Rocksalt $\mathrm{O}_{h}{ }^{5}-\mathrm{Fm} 3 m$ ”, Phys. Rev. 170, 639 (1968).

[33] R. Schönemann, N. Aryal, Q. Zhou, Y.-C. Chiu, K.-W. Chen, T. J. Martin, G. T. McCandless, J. Y. Chan, E. Manousakis, and L. Balicas, Fermi Surface of the Weyl Type-II Metallic Candidate $\mathrm{WP}_{2}$, Phys. Rev. B 96, 121108(R) (2017).

[34] M.-Y. Yao, N. Xu, Q. S. Wu, G. Autès, N. Kumar, V. N. Strocov, N. C. Plumb, M. Radovic, O. V. Yazyev, C. Felser, J. Mesot, and M. Shi, Observation of Weyl Nodes in Robust Type-II Weyl Semimetal $\mathrm{WP}_{2}$, Phys. Rev. Lett. 122, 176402 (2019).

[35] L. Lindsay, D. A. Broido, and T. L. Reinecke, FirstPrinciples Determination of Ultrahigh Thermal Conductivity of Boron Arsenide: A Competitor for Diamond?, Phys. Rev. Lett. 111, 025901 (2013).

[36] Y. Tian, A. A. Reijnders, G. B. Osterhoudt, I. Valmianski, J. G. Ramirez, C. Urban, R. Zhong, J. Schneeloch, G. Gu, I. Henslee, and K. S. Burch, Low Vibration High Numerical 
Aperture Automated Variable Temperature Raman Microscope, Rev. Sci. Instrum. 87, 043105 (2016).

[37] Y. Wang, G. B. Osterhoudt, Y. Tian, P. Lampen-Kelley, A. Banerjee, T. Goldstein, J. Yan, J. Knolle, H. Ji, R. J. Cava, J. Nasu, Y. Motome, S. E. Nagler, D. Mandrus, and K. S. Burch, The Range of Non-Kitaev Terms and Fractional Particles in $\alpha-\mathrm{RuCl}_{3}$, npj Quantum Mater. 5, 14 (2020).

[38] P. Giannozzi et al., QUANTUM ESPRESSO: A Modular and Open-Source Software Project for Quantum Simulations of Materials, J. Phys. Condens. Matter 21, 395502 (2009).

[39] P. Giannozzi et al., Advanced capabilities for materials modelling with QUANTUM ESPRESSO, J. Phys. Condens. Matter 29, 465901 (2017).

[40] D. R. Hamann, Optimized Norm-Conserving Vanderbilt Pseudopotentials, Phys. Rev. B 88, 085117 (2013).

[41] J. P. Perdew, K. Burke, and M. Ernzerhof, Generalized Gradient Approximation Made Simple, Phys. Rev. Lett. 77, 3865 (1996).

[42] S. Poncé, E. Margine, C. Verdi, and F. Giustino, EPW: Electron-Phonon Coupling, Transport and Superconducting Properties Using Maximally Localized Wannier Functions, Comput. Phys. Commun. 209, 116 (2016).
[43] N. Marzari and D. Vanderbilt, Maximally Localized Generalized Wannier Functions for Composite Energy Bands, Phys. Rev. B 56, 12847 (1997).

[44] I. Souza, N. Marzari, and D. Vanderbilt, Maximally Localized Wannier Functions for Entangled Energy Bands, Phys. Rev. B 65, 035109 (2001).

[45] R. Sundararaman, K. Letchworth-Weaver, K. A. Schwarz, D. Gunceler, O. Yalcin, and T. Arias, JDFTx: Software for Joint Density-Functional Theory, SoftwareX 6, 278 (2017).

[46] A. Dal Corso, Pseudopotentials Periodic Table: From $H$ to Pu, Comput. Mater. Sci. 95, 337 (2014).

[47] A. M. Rappe, K. M. Rabe, E. Kaxiras, and J. D. Joannopoulos, Optimized Pseudopotentials, Phys. Rev. B 41, 1227 (1990).

[48] J. P. Perdew, A. Ruzsinszky, G. I. Csonka, O. A. Vydrov, G. E. Scuseria, L. A. Constantin, X. Zhou, and K. Burke, Restoring the Density-Gradient Expansion for Exchange in Solids and Surfaces, Phys. Rev. Lett. 100, 136406 (2008).

[49] F. Giustino, M. L. Cohen, and S. G. Louie, Electron-Phonon Interaction Using Wannier Functions, Phys. Rev. B 76, 165108 (2007). 